\title{
Decision Making Systems for Managing Business Processes in Enterprises Groups
}

\author{
Ali F. Dalain \\ Human Resources Management Department \\ University of Jeddah, College of Business, KSA
}

\begin{abstract}
In the current economic realities, the forms of integration business entities through the creation of enterprise groups (EGs), reorganized from industry structures or created a new by acquiring existing companies, are becoming increasingly relevant. The economic activity of the enterprise is carried out in the conditions of economic instability and improvement of the system economic relations, which imposes fundamentally new requirements in the sphere of managing the interaction of enterprises. Under these conditions, the successful development of the enterprises and often their very existence depend both on the effective use of the management systems themselves and on the competence of the management decisions made. Consequently, for decision makers and managers of Group Policy (GP), the problem of evaluating the development of GP and promptly making sound management decisions in an unstable and rapidly changing economic environment is considered a particular relevance. One of the promising ways to solve this problem is the development of decision support systems (DSS), using scientifically based decision-making methods based on modern mathematical apparatus and computer equipment. At present, the approach to managing the development of the EGs is associated with the representation of the latter as a multi-agent system (MAS). The DSS does not replace, but complements the existing management systems in the EGs, interacting with them, and uses in its work information about the functioning of EGs units.
\end{abstract}

Keywords-Management systems; decision support systems; multi-agent systems; group policy; enterprise groups

\section{INTRODUCTION}

At all stages of the economic development of EGs, the most important problem in the activities of enterprises is the problem of increasing the competitiveness of their products, which can be achieved both by modernizing production and by optimizing the interaction of enterprises in EGs [1],[2]. One of the priorities is the development and use of DSS in the management of business processes EGs.

Under the management of business processes we can smoothly understand the system of targeted impacts, in which, by means of decision-makers, decision makers implement measures to improve the efficiency of the EGs. Several types of impacts in this research are considered: the selection and implementation of investment projects (IP) [3], ensuring the production of competitive products; optimizations of the system parameters interaction with agents in MAS for the manufacture and sale of products; restructuring of EGs units [4], [5].
The survival of industrial enterprises in conditions of economic instability often directly depends on a successful management of business processes. At the same time, considerable difficulties appear in the EGs already at the initial stage of modernization of management [6]. This is due to the fact that most of the existing traditional software for managing the development of enterprises are built on the classical principles of budgeting - control and are not sufficiently effective at present to manage the business processes of EGs. In addition, the transition from the designated strategy for the development of EGs to specific actions of the performers is sometimes difficult to implement due to the lack of a regular unified mechanism that would set the necessary priorities, allow preparing and evaluating solutions, analyzing the distribution of IP across EGs [7], and controlling the consistency and efficiency of execution in IP and also supported the possibility of joint decision-making on a number of current issues [8].

However, it should be noted that the state of affairs in the field of DSS applications to ensure the effectiveness of business process management does not sufficiently meet the needs of the enterprise in modern conditions, and there are a number of scientific problems that require systemic solutions [9]. Among them, it can be noted: the need to develop a decision-making methodology in determining the optimal control regimes for the interaction of industrial enterprises in the EGs; the need to develop the principles of information and analytical support for DSS when optimizing the management systems of a EGs [10], the lack of common models for selecting and implementing IPs in several EGs under the direction of the managing company (MC). Therefore, the development and applications of DSS in the management of business processes and EGs is currently a pressing and timely task.

The aim of the work is a systematic analysis of information processes for managing the activities of EGs, the development of DSS to increase the efficiency of the implementation in IP, as well as the optimization of teamwork (interaction) of enterprises belonging to the EGs. To achieve these goals, the main research methods used were: system analysis, automatic control theory, decision theory, structural and dynamic analysis, system modeling, numerical methods, nonlinear programming [9].

Practical values of this research are considered the applications of the developed models in the management system of joint business processes in EGs allows taking into account the change in the performance indicators of each 
industrial enterprises over time of their joint work [7],[11]. This makes it possible to conduct an analysis of the stability in the functioning of EGs and to take control decisions at the optimal points in time to obtain maximum values of the functional quality of each enterprise [12].

\section{RESEARCH FRAMEWORK}

Modern concepts of managing complex distributed systems in various industries are based on the man-machine organization of management processes, in which the role of decision-making is assigned to a person, and the machine provides information support for the stages of generating and generating alternative solutions [13]. It is noted that the use of this approach is a necessary measure to overcome a high level of uncertainty in the formulation and conditions for solving problems of managing complex, nonlinear and dynamic objects, such as EGs. At the same time, the effectiveness of the control systems of such EGs is largely determined by the subjective properties of the decision maker (DM) operating in the control loop, which in turn requires it to have a high level of competence both in managing business processes of the EGs and in the resulting problem situations [14],[15].

Currently known and widely used method of overcoming subjectivity and increasing the level of competence of decision makers is the use of DSS. DSS provides the correctness of solving problems by choosing rational options for managing business processes through the integrated use of a set of technologically interconnected services that implement traditional and advanced information technologies.

DSS can be represented as a set of management subsystems operating in the information environment in accordance with established information flow procedures, which determine the sequence of business process implementation steps, as well as methods of transmitting, storing and processing information in the management process [16],[17]. Consequently, one of the main conditions for the effective functioning of the DSS is a constant analysis and processing, establishment of links and ways of circulating information flows between information sources and receivers, which provides an integrated approach to the development and implementation of management decision options for decision makers.

For management of enterprises known and wellestablished in practice is an approach based on the use of multi-agent DSS, which combines various methods of analyzing situations and forming management decisions implemented by agents of MAS [9]. It is shown that multiagent technologies can be considered as the basis for creating effective methods and tools for developing business process management systems. The issues of creation and application of DSS in situations related to the optimization of the interaction between agents in the network structures of the MAS and hierarchical structures are considered. The most important components of such systems, which include subsystems for modeling business processes, are highlighted [18].

An analytical review of the DSS structures and information processing methods in them is given. The most popular information processing systems, their functionality, architecture, software platforms, design principles, etc. is considered. The analysis of preliminary data preparation systems for making management decisions based on ERP, IDEF, MRP, ERPII standards was carried out. The advantages and disadvantages of replicable systems of this class are noted, including the complexity, duration and cost of implementation, and examples of implemented DSS for various fields of activity are given. It was concluded that there are no systems applicable for the purposes of managing the business processes of the EGs, taking into account the optimization of the modes of cooperation between the MAS agents [19],[20].

In the next stage of this research, business process management models are developed based on the selection and implementation of IP in DSS. The proposed management models are conceptual in nature and reflect the most significant aspects of the joint work of the EGs [6],[14]. In this case, the main focus is on determining the basic laws of management processes, as well as the trends and strategies for their development, depending on the parameters of the work of the EGs between themselves and the external environment.

The analysis of the MAS works, consisting of industrial enterprises is under the control of the Criminal Code. It is shown that each enterprise that is included in the EGs can be represented as an independent agent acting within the limits of the powers assigned to it. From the position of system analysis, such MAS can be represented by several classes: the central management company, territorial management companies, industrial enterprises (agents A, B, C) (Fig. 1).

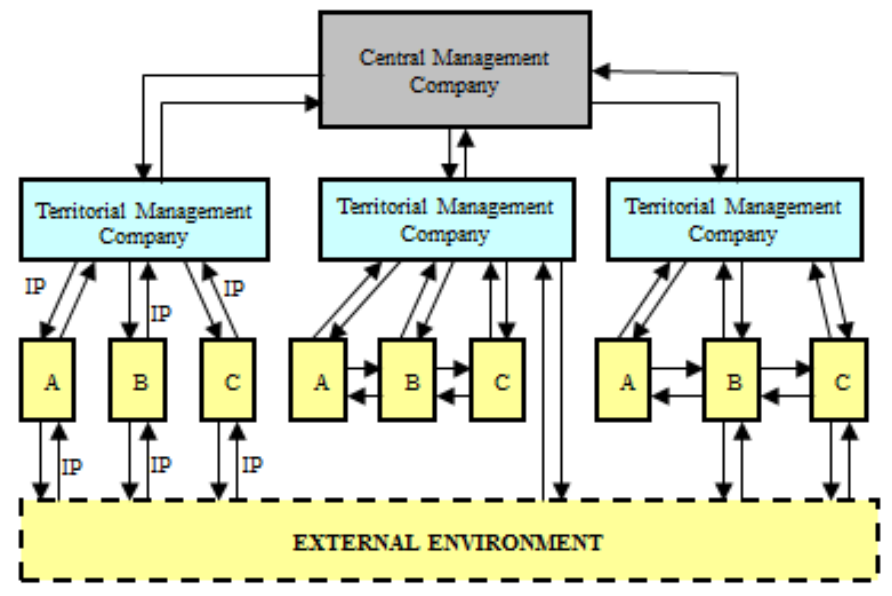

Fig. 1. Scheme of Industrial Enterprises Group.

\section{PROPOSED METHOdOLOGY AND EXPERIMENTAL RESULTS}

In solving the problems of managing business processes of the implementation of IP, the Solow single-product model is used, which can be applied both to a separate industrial enterprise and to the entire EGs. In addition, it is assumed that the MAS operates in the established external environment, whereas, there is no time lag between investments and their development, also the pace of enterprise development is characterized by the dynamics of production assets, which in 
turn is determined by the amount of investment resources (deductions from profits or external investments).

When optimizing the management of IP implementation at an industrial enterprise and the expense of external sources, it is assumed that at the initial moment of time the level of the fixed production assets of the enterprise in monetary terms is $\mathrm{X} 0$, and in order to further develop the enterprise, it is required to increase it to the required value of XT due to foreign investment of the agent investor over time $[0, \mathrm{~T}]$. The dynamics of changes in the current level of cost for production assets of the enterprise in this case can be written in the following form:

$$
X^{\prime}(t)=-\mu X(t)+D(t) .
$$

Here $\mu$ is the equipment depreciation factor, and $D(t)$ is the investment rate (the investor's control parameter). An investor, when participating in an IP, tries to control the parameter $D(t)$ in such a way as to obtain the minimum values of its own total investment costs $G(T)$ (functional quality) $G(T)=\int_{0}^{T} D(t) e^{-\lambda t} d t \longrightarrow \min$,

where $\lambda$ is the discount rate of financial flows.

To determine the optimal investment process, it is necessary to integrate equation (1) taking into account the conditions for $G(T)$. If the investment is constant $\left(D(t)=D_{0}=\right.$ const $)$, the solution to this problem can be obtained in the following form: $G_{0}=D_{0}\left(1-e^{-\lambda T}\right) / \lambda$. From this expression it is clear that the total investment costs depend linearly on the rate of investment. Since the linear function has no extremes, it is not possible to optimize the investment process. To optimize the investment process, instead of linear, we will use a quadratic function, and then the investor quality functional will have the following form:

$$
G_{1}=\int_{0}^{T} D^{2}(t) e^{-\lambda t} d t \longrightarrow \min .
$$

For this variant of investor's work, it is also necessary to integrate equation (1) taking into account the conditions for G1. Such a task belongs to the class of Lagrange problems of optimal control theory, and for its solution one can use the Pontryagin maximum principle.

To solve the problem in this way, the Hamilton function is compiled, and the expressions for $D^{*}(t)$ are found for which the Hamiltonian will have the maximum value. After integration (1), taking into account the requirements for $G_{1}$ and the boundary values $\left(X_{0}\right.$ and $\left.X_{T}\right)$, the following trajectory of controlling the rate of investment was determined:

$$
D^{*}(t)=\left(X_{T}-X_{0} e^{-\mu T}\right)(\mu+\theta) e^{\theta t} /\left(e^{\theta T}-e^{-\mu T}\right)
$$

Where $\theta=\mu+\lambda$, based on the obtained expressions for $D^{*}(t)$ and $D_{0}$, the total costs of the investor were calculated using the expression for $G(T)$.

The calculations have shown that managing the investment process with $D^{*}(t)$ allows the investor to reduce his total costs while achieving the same values of $X_{\mathrm{T}}$. Table I shows the decrease in total investor costs (as a percentage) when financing for $D^{*}(t)$ as compared to the rate of financing for $D_{0}=$ const for a number of values of $\mu$ and $\lambda$ for $X_{T} / X_{0}=2$ and $T=2$.

Thus, the management of the investment process according to the obtained expression $D^{*}(t)$ compared to $D_{0}=$ const can reduce the total investor costs when the same values of $X_{T}$ are reached.

Also in this research discusses such IPs, when an industrial enterprise additionally uses its profits with selling an IP, which it invests in investments or in its own savings. The choice of one of these options is made by the company from the conditions for obtaining maximum profit. The investor participates in joint work by acquiring shares on which he receives dividends. The equation of agents working together in such MAS, by analogy with (1), will be:

$$
X^{\prime}(t)=-\mu X(t)+v \pi+\xi D(t) \text {. }
$$

Here $\pi$ is the profit, and $v$ and $\xi$ are the control parameters of the enterprise and the investor. It is assumed that when $v=1$, the profit of the enterprise goes to them for investment, and when $v=0$, it goes into its own savings. Similarly, when $\xi=1$, investments are made, and when $\xi=0$, there is no investment. Additionally, there is the following restriction: $v$ and $\xi$ must vary in the range $[0,1]$. At the same time, an instant investment is taken, the analysis uses a single-factor linear production function, and the change in the company's profit relative to fixed assets also has a linear relationship.

The objective function of an enterprise $\left(G_{l m}\right)$ is both to increase its own savings and to increase the value of its fixed assets, and the objective function of an investor $\left(G_{2}\right)$ is to get maximum profit:

$$
\begin{aligned}
& G_{1 m}=\int_{0}^{T} \pi(1-v) e^{-\lambda t} d t+X(T) \rightarrow \max , \\
& G_{2}=\int_{0}^{T}(\alpha \pi L-\xi D) e^{-\lambda t} d t \rightarrow \max
\end{aligned}
$$

Here $\alpha$ is the share of the profit directed by the enterprise to pay dividends on its shares, and $L$ is the share of shares owned by the investor from the total amount on which dividends are paid.

TABLE. I. COST REDUCTION (\%) OF INVESTMENT AT D(T) COMPARED WITH D0

\begin{tabular}{|l|l|l|l|l|l|l|}
\hline & $\lambda=0.05$ & $\lambda=0.10$ & $\lambda=0.15$ & $\lambda=0.20$ & $\lambda=0.25$ & $\lambda=0.30$ \\
\hline$\mu=0.05$ & 0.7 & 0.8 & 1.2 & 2.0 & 3.0 & 4.1 \\
\hline$\mu=0.10$ & 1.1 & 1.6 & 2.3 & 3.2 & 4.4 & 5.9 \\
\hline$\mu=0.15$ & 1.6 & 2.1 & 3.2 & 4.5 & 5.7 & 8.0 \\
\hline$\mu=0.20$ & 2.3 & 3.3 & 4.5 & 5.7 & 7.3 & 9.6 \\
\hline$\mu=0.25$ & 3.3 & 4.3 & 5.6 & 7.3 & 8.9 & 11.1 \\
\hline
\end{tabular}


After analyzing the task, it was obtained that for integrating equation (3) with due regard for requirements (4), Pontryagin's maximum principle can be used, which allows one to obtain optimal trajectories of control parameters $v$ and $\xi$ in the presence of restrictions on them. As a result of solving the problem, it was obtained that over time of joint work [0, $\mathrm{T}]$, the control parameters $v$ and $\xi$ should change as follows:

$$
v=\left\{\begin{array}{lll}
1 & \text { if } & t>t_{0} ; \\
0 & \text { if } & t<t_{0} ;
\end{array} \quad \xi=\left\{\begin{array}{lll}
1 & \text { if } & t<t_{1} ; \\
0 & \text { if } & t_{1}<t<t_{0} .
\end{array} \quad \xi=\left\{\begin{array}{lll}
1 & \text { if } & t_{0}<t<t_{2} ; \\
0 & \text { if } & t>t_{2} .
\end{array}\right.\right.\right.
$$

As can be seen, changes in the control parameters are of a relay nature and equal to one of their two possible limit values. The moments of time at which changes in these parameters should occur are equal to:

$$
\begin{aligned}
t_{0} & =T \frac{b-\mu}{b-\theta} ; \quad t_{1}=t_{0}+\frac{1}{\theta} \ln \frac{b L-\theta}{b L} \\
t_{2} & =T+\frac{1}{\theta} \ln \frac{\alpha b L-\theta}{\alpha b L}
\end{aligned}
$$

$$
\text { Where } \theta=\mu+\lambda \quad, \quad b=\partial \pi / \partial X, \quad \text { wherein }
$$
$t_{1}<t_{0}<t_{2}<T$.

In order to fulfill conditions (4), the optimal management of the use of its profits by an enterprise will be as follows. On the time interval $\left[0, \mathrm{t}_{0}\right]$, where $v(t)=0$, the profit of the enterprise is sent to its own savings, and on the time interval $\left[\mathrm{t}_{0}, \mathrm{~T}\right]$, where $v(t)=1$, it is spent on the IP (Fig. 2 ).

For optimal investment management, the investor (Fig. 2) acquires shares at the time interval $[0, \mathrm{t} 1]$, receiving additional dividends, at the time $t 1$ stops buying shares $(\xi=0)$ and only receives dividends at $[\mathrm{t} 1, \mathrm{t} 0]$ on previously acquired shares. If the company in the time interval $[\mathrm{t} 0, \mathrm{~T}]$ does not pay dividends $(\alpha=0)$, then from the moment of time to the investor stops his participation in the joint IP. If during the time period $[\mathrm{t} 0, \mathrm{~T}]$ the company continues to pay dividends, the investor starts buying shares again before the time $t 2$. At this point in time, the acquisition of shares is terminated, and in the period $[\mathrm{t} 2, \mathrm{~T}]$, the investor will only receive dividends on previously acquired shares. Such management of joint work of the enterprise and the investor allows obtaining the maximum values of the functional quality $(\mathrm{G} 1 \mathrm{~m}$ and $\mathrm{G} 2)$ to all participants of the implementation of the IP.

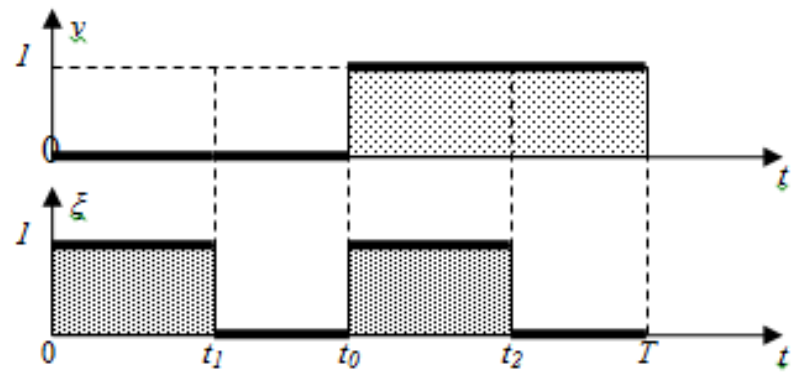

Fig. 2. Optimal Change of Control Parameters Enterprises (v) and Investor $(\xi)$
In the next stage of the research, optimization of the management of the joint venture for the manufacture and sale of industrial products is carried out. It discusses the work of the MAS, consisting of several agents that are enterprises of EGs, for example, auxiliary and main production (Fig. 3). The auxiliary production enterprise (agent A) acquires the necessary materials and components, conducts their control, preprocessing and then transfers them to the main production for manufacture, testing, assembly and further implementation (agents B and C). The joint work of agents over time in such MAS can be represented by the following system of differential equations:

$$
\left\{\begin{array}{l}
x^{\prime}=U(X 1-x)-V(Z 1-z)-R(Y 1-y) \\
z^{\prime}=V(Z 1-z)-W z \\
y^{\prime}=R(Y 1-y)-S y .
\end{array}\right.
$$

Here $x, y$, and $z$ accordingly, the quantity of products in the warehouse of agents $\mathrm{A}, \mathrm{B}$ and $\mathrm{C}, U(t)$ is the speed of production by agent $\mathrm{A}, V(t), W(t), R(t), S(t)$ the speed of the rate acquired and subsequently sold by agents $\mathrm{B}$ and $\mathrm{C}$ units of production, $X 1, Z 1$ and $Y 1$ are the maximum production capacities of the warehouses of agents A, B and C.

During the joint work $[0, T]$, each of the agents seeks to get the maximum profit for themselves ( $J 1$ for agent $\mathrm{A}, J 2$ and $J 3$ for agents $\mathrm{B}$ and $\mathrm{C}$ ):

$J_{1}=\int_{0}^{T}\left[c_{1} V(Z 1-z)+c_{3} R(Y 1-y)-c_{0} U(X 1-x)-d_{1} x\right] d t \rightarrow \max$

$J_{2}=\int_{0}^{T}\left[c_{2} W z-c_{1} V(Z 1-z)-d_{2} z\right] d t \rightarrow \max$

$J_{3}=\int_{0}^{T}\left[c_{4} S y-c_{3} R(Y 1-y)-d_{3} y\right] d t \rightarrow \max$

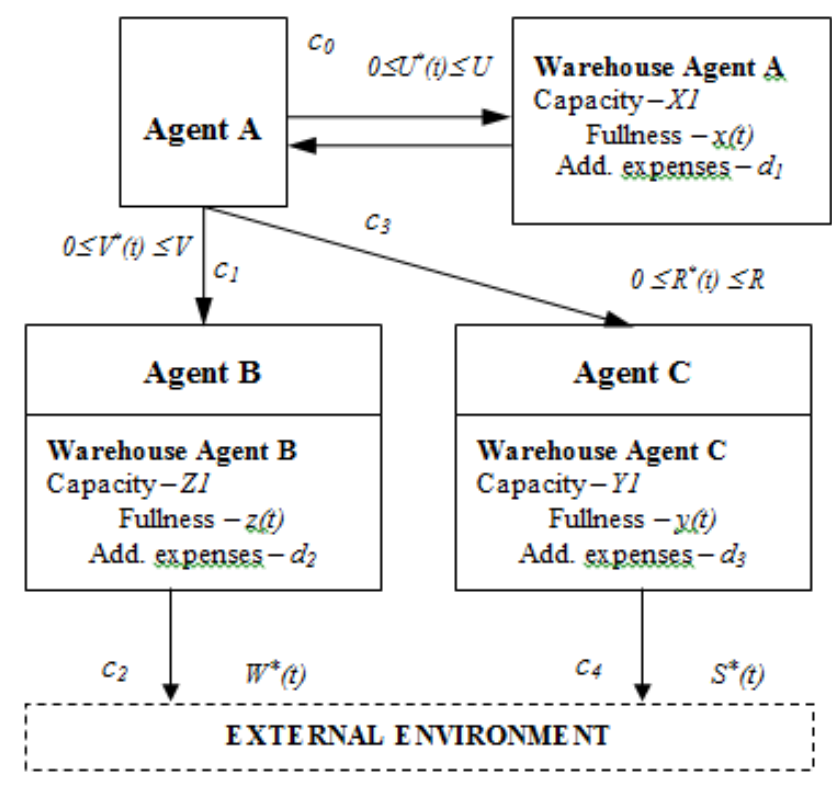

Fig. 3. Collaboration of EGs Agents. 
where $c_{0}, c_{1}, c_{2}, c_{3}, c_{4}$ are the unit costs manufactured by agent $\mathrm{A}$, the products purchased and sold by agents $\mathrm{B}$ and $\mathrm{C}$, and $d_{1}, d_{2}$ and $d_{3}$ are the additional costs of transporting and storing them in the agents' warehouses.

Each agent can get the maximum value of its own functional quality by changing its control parameters $U(t)$ (agent A), $V(t), W(t)$ (agent B) and $R(t), S(t)$ (agent $\mathrm{C}$ ).

Consider first the work of agent $B$ in such MAS. To do this, it is necessary to integrate (5) taking into account the fulfillment of the requirements (8). In solving this problem, we will use the Pontryagin maximum principle, for this we construct the Hamilton function:

$$
H_{3}=\psi_{1} U(X 1-x)+h_{1} R(Y 1-y)+h_{2} S y+h_{3} V(Z 1-z)-d_{3} y,
$$

Where a $\psi 1, \psi 2$ and $\psi 3$ are auxiliary variables defined by the expressions:

$\psi_{1}^{\prime}=-\frac{\partial H_{3}}{\partial x}=U \psi_{1} ; \psi_{2}^{\prime}=-\frac{\partial H_{3}}{\partial z}=V h_{3}+W \psi_{2} ; \psi_{3}^{\prime}=-\frac{\partial H_{3}}{\partial y}=R h_{1}-S h_{2}+d_{3}$

In accordance with the Pontryagin maximum principle, the optimal control of the work of agent $\mathrm{C}$ will be if the Hamilton function has the maximum value. This will be the case if the control parameters of agent $\mathrm{C}$ are changed as follows:

$$
R^{*}(t)=\left\{\begin{array}{ll}
0, & h_{1}<0 ; \\
R, & h_{1}>0 ;
\end{array} \quad S^{*}(t)= \begin{cases}0, & h_{2}<0 \\
S, & h_{2}>0\end{cases}\right.
$$

It can be seen that the changes in the control parameters of the agent $\mathrm{C}$ are of a relay nature and are equal in magnitude to one of their two possible limiting values. Moreover, the whole process of changing these parameters consists of two intervals, in which these parameters have a constant value, and the duration of these intervals is determined by the auxiliary variables $\psi 1, \psi 2$ and $\psi 3$, which can be found from the solution of the differential equations for them. The boundary values for these variables were defined in this part of the research, and they are respectively equal: $\psi_{l}(T)=\psi_{2}(T)=\psi_{3}(T)=0$.

The expressions for auxiliary variables obtained in this chapter showed that the function $h_{2}>0$ on the entire interval $[0, \mathrm{~T}]$, the function $h_{1}>0$ on the interval $\left[0, \mathrm{t}_{2}\right]$, and on the interval $\left[\mathrm{t}_{2}, \mathrm{~T}\right]$ the function $h_{1}<0$. The time $\mathrm{t}_{2}$, at which the control parameter $R^{*}(t)$ becomes zero, and agent $\mathrm{C}$ stops purchasing products from agent $\mathrm{A}$, is equal to:

$t_{2}=T+\frac{1}{S} \ln \frac{\left(c_{4}-c_{3}\right) S-d_{3}}{c_{4} S-d_{3}} ; \quad t_{1}=T+\frac{1}{W} \ln \frac{\left(c_{2}-c_{1}\right) W-d_{2}}{c_{2} W-d_{2}}$

The work of Agent B is considered in a similar way. It has been obtained that the optimal control of the process of his work will be the same as for Agent $\mathrm{C}$, i.e. at the initial time interval $\left[0, \mathrm{t}_{1}\right]$, he acquires, and at the time interval $\left[\mathrm{t}_{1}, \mathrm{~T}\right]$ stops purchasing products from agent A. The value of time $t_{l}$, at which its control parameter $V^{*}(t)$ changes, is determined by 9).

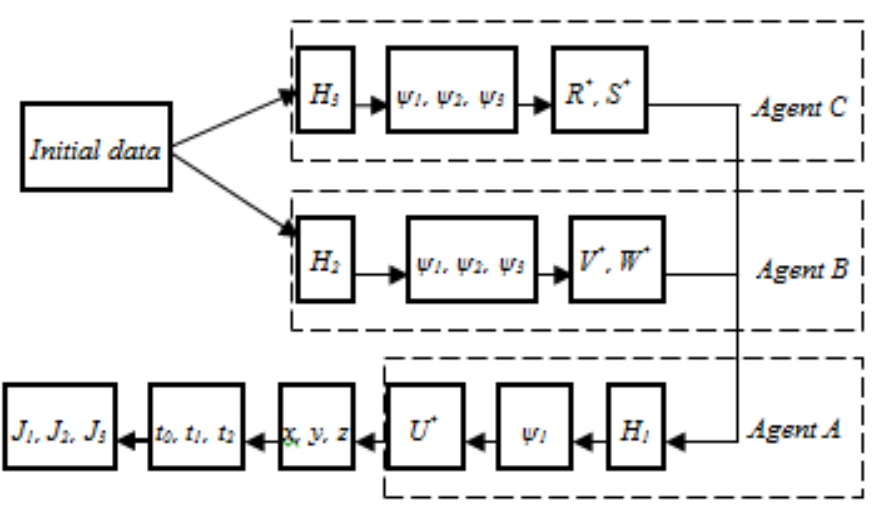

Fig. 4. Algorithm for Determining the Optimal Parameters for Managing Agents.

The determination of the optimal modes of operation for agent $\mathrm{A}$ was also carried out using the Pontryagin maximum principle, using the system of equations (5) and (6). As a result of the analysis, it was determined that in order to obtain maximum profit, Agent A should produce products in the initial period of time $\left[0, \mathrm{t}_{0}\right]$, and stop production in the period of time $\left[\mathrm{t}_{0}, \mathrm{~T}\right]$. Fig. 4 shows the scheme for determining the control parameters of agents $\mathrm{A}, \mathrm{B}$ and $\mathrm{C}$ when they work together in the MAS.

When determining the point in time $t_{0}$, it is necessary to take into account that Agent A usually tries to fully realize all products manufactured for them, i.e. receive $x(T)=0$. In this stage of the research, an expression is obtained for determining the quantity of products in the warehouse of agent $\mathrm{A}$ at the end of the collaboration time:

$x(T)=K_{0}+K_{1} x_{0}+K_{2}\left(t_{1}-t_{0}\right)+K_{3}\left(e^{-(V+W) t_{1}}-e^{-(V+W) t_{0}}\right)$

Where $\mathrm{K}_{0}, \mathrm{~K}_{1}, \mathrm{~K}_{2}$ and $\mathrm{K}_{3}$ are constant values depending on the initial indicators of the joint work of agents. Equating expression (10) to zero, we can find the unknown value $t_{0}$. Due to the fact that the resulting equation is non-linear, its solution is carried out by numerical methods. For this purpose, a program is compiled, the algorithm of which is shown in Fig. 5. Its input data are the left $(a)$ and right $(b)$ boundaries of the interval in which the desired one $\left(t_{0}\right)$ is located, as well as the accuracy of calculations $(\xi)$.

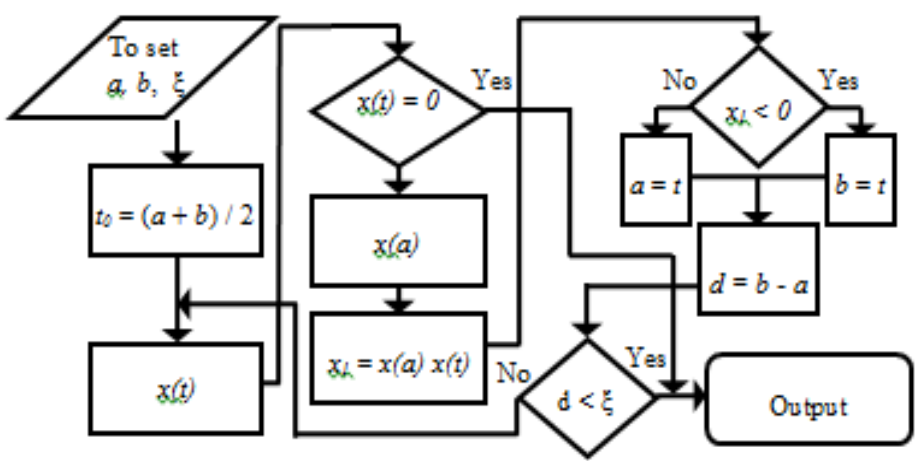

Fig. 5. Algorithm for Determining the Optimal Moment of Time $t_{0}$ Stopping Production of the Agent A. 
The optimal process for managing the joint work of agents in such MAS will be as follows. On the time interval $[0, \mathrm{t} 0]$, agent $\mathrm{A}$ makes at time $\mathrm{t} 0$, stops production and does not make production on the interval $[\mathrm{t} 0, \mathrm{~T}]$. Agent $\mathrm{B}$ in the time interval $[0, \mathrm{t} 1]$, and agent $\mathrm{C}$ in the interval $[0, \mathrm{t} 2]$ are purchasing products. At time $\mathrm{t} 2$ agent $\mathrm{C}$ and at time $\mathrm{t} 1$ agent $\mathrm{B}$ is stopping buying products from agent $\mathrm{A}$. At the same time, agents $\mathrm{B}$ and $\mathrm{C}$ are engaged in selling products throughout the entire time interval $[0, \mathrm{~T}]$.

The resulting collaboration scheme has a rationale - it is not profitable for any of the agents to produce (acquire) excess products, which subsequently cannot be fully realized. Such a scheme of parallel collaboration of agents does not always suit Agent A, since there are periods of time during which its production will stand idle. To eliminate this drawback, a sequential scheme of agents is proposed. For the case of the MAS of the three agents A, B and C, it is shown in Fig. 6.

Initially, agent $\mathrm{A}$ works with agent $\mathrm{B}$, at time t0 stops the production of products for agent $\mathrm{B}$, and instead of completely stopping his production, he begins to manufacture products for agent $\mathrm{C}$ during a period of time $[\mathrm{t} 0, \mathrm{~T}]$. From the moment of time $\mathrm{T}$, work with agent $\mathrm{B}$ begins again and then the whole cycle of joint work of agents $\mathrm{A}, \mathrm{B}$ and $\mathrm{C}$ is repeated again. With such a consistent scheme, all agents (A, B, and C) are excluded from work stoppages.

Fig. 7 shows the change in total profit of agent $\mathrm{A}(\mathrm{JA}=\mathrm{JB}$ $+\mathrm{JC}$ ) over time $\mathrm{t}$ with the above sequential work pattern. Curves JB (agent B) and JC (agent C) show the change in the profit of agent $A$ in the event that he works separately with each of these agents. The sequential scheme of work of agent $\mathrm{A}$ is that from the initial moment to $\mathrm{t} 0=8.0$ he works with agent $\mathrm{C}$, and his profit is negative, i.e. the cost of manufacturing is still more than the cost of the products sold to them. From the moment $\mathrm{t} 0=8.0$, he ceases to manufacture products for agent $\mathrm{C}$, and only sells to him previously manufactured products. Simultaneously, the production of products for agent $\mathrm{B}$ begins, which continues until time $\mathrm{T}=$ 20.0. From the time point $\mathrm{T}=20.0$, work with agent $\mathrm{C}$ begins again, and the whole cycle repeats again.

The moments of time $\mathrm{t} 0, \mathrm{t} 1$ and $\mathrm{t} 2$ depend on the initial parameters values for the joint work of agents. At the stage of discussing the conditions of joint work of agents, decision makers on the basis of the recommendations DSS agree on the values of these parameters that ensure the continuous operation of each agent.

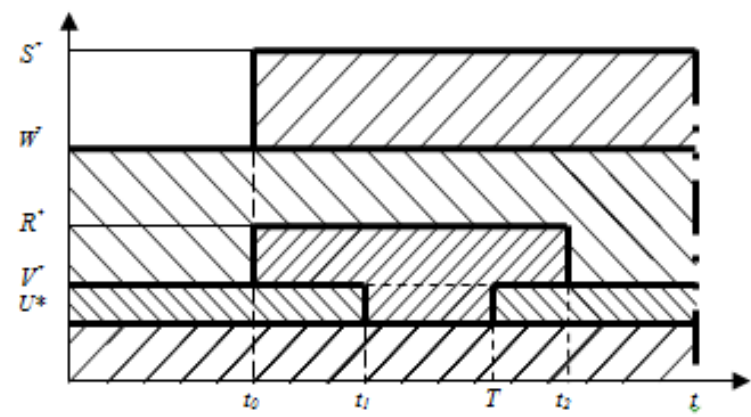

Fig. 6. Change of Control Parameters of Agents A, B and C During their Sequential Work.

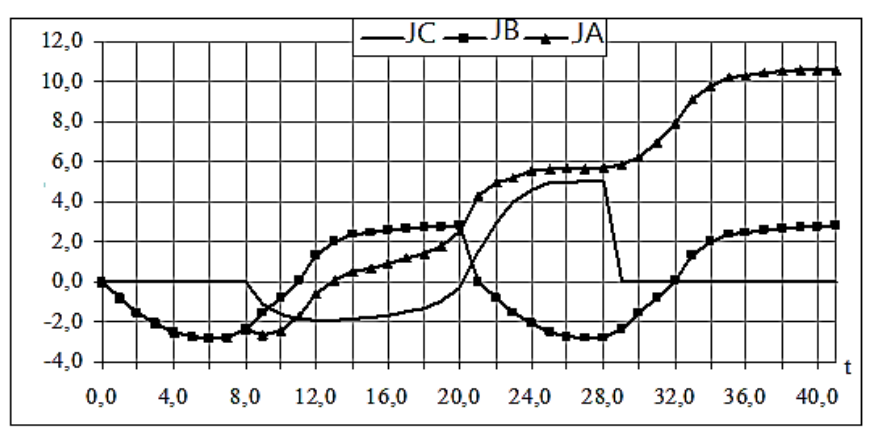

Fig. 7. Sequential Work of Agents A, B and C.

\section{RESULTS AND PRACTICAL IMPLEMENTATIONS}

In practice, the joint work of manufacturers and consumers of products may differ from that discussed above. In the annex to the research, additional options for the joint work of agents are given, which differ from each other in the initial conditions that characterize the manufacture, acquisition and sale of products. So, for example, the joint work of the MAS of two agents - the manufacturer of the product (agent A) and his dealer (agent B) can be conditionally represented by the following system of equations:

$\left\{\begin{array}{l}x^{\prime}=U-V x \\ z^{\prime}=V x-W z .\end{array}\right.$

The functional quality ( $\mathrm{J} 1$ of agent $\mathrm{A}, \mathrm{J} 2$ of agent $\mathrm{B}$ ) for this variant of their joint work will be as follows:

$$
J_{1}=\int_{0}^{T}\left(c_{1} V x-U c_{0}-d_{1} x\right) d t-c_{1} x(T) \rightarrow \max
$$

$$
J_{2}=\int_{0}^{T}\left(c_{2} W z-c_{1} V x-d_{2} z\right) d t-c_{2} z(T) \rightarrow \max
$$

The task for both agents is to search for the optimal trajectories of changes in the control parameters $U^{*}, V^{*}$ and $W^{*}$, at which $J 1$ and $J 2$ will have maximum values. In this stage of this research, we analyze the operation of such a system and find the optimal modes for changing the control parameters of agents $\mathrm{A}$ and $\mathrm{B}$, which ensure maximum efficiency for each of them.

Due to the unstable modern market situation, there are cases when one of the agents at some point in time decides to stop working together. In this case, he needs to fully realize all his products. This part of the research examines such possible cases and determines the minimum possible sales times, which are equal to (T1 and T2) for agents $\mathrm{A}$ and $\mathrm{B}$ :

$$
T_{1}=-\frac{1}{V} \ln \frac{V_{1}}{V_{1}+V x_{k}} ; \quad T_{2}=-\frac{1}{W} \ln \frac{w}{w+W z_{k}}
$$

Here $x_{k}$ and $z_{k}$ - the number of products in the warehouses of agents at the time of the decision to cease 
collaboration, $V 1$ and $w$, respectively, the minimum rate of acquisition and sale of products by agent $B$.

We provide examples of determining the optimal processes for managing the joint work of agents in MAS, and also discuss the practical implementation of the developed DSS in the management of EGs business processes.

One of the mandatory conditions for the joint work of agents is to obtain the maximum values of their functional quality by each of them. From the expressions for these functional quality $(6-8,11-12)$ it can be seen that their values depend on many initial indicators. Fig. 8 shows the typical nature of the change over time $t$ of the joint operation of the $J_{2}$ functional of agent $\mathrm{B}$ depending on the $\cos t c_{l}$ of the product it purchases from agent $\mathrm{A}$ with the following initial data of joint work of agents: $T=20.0 ; c_{0}=1.0 ; c_{2}=2.5$. It can be seen that the change in $J_{2}$ from $c_{1}$ and $t$ has a significant non-linear dependence, with the maximum value of $J_{2}(T)$ being $c_{1} \approx 1.3-1.5$.

The same character affects $c_{l}$ and $t$ on the change in the functional quality of agent $\mathrm{A}$, while its maximum value will be at a different value of $c_{1}$. The choice of a specific value $c_{1}$ should be made by the agents on the basis of the recommendations of DSS when coordinating the source data and modes of their joint work.

Also depending on the practical implementation, the graphs and tables show the effect of the initial indicators on the change in the control parameters of the agents, on the basis of which solutions for managing MAS agents are developed in DSS. Fig. 8 shows the dependence of the functional $J_{2}$ on $\mathrm{t}$ and $c_{1}$.

The maximum values of the functional quality of each agent are largely determined by the initial values of the collaboration indicators. Each of the agents usually seeks to select them such that would provide him the greatest profit. To determine the values of such indicators, it is necessary to optimize the profit margin relative to possible changes in the values of the initial parameters. To this end, it is proposed to use numerical methods for finding the maximum function of several variables.

This step analyzes the existing search methods and shows that in the DSS one can use the deformable polyhedron method. A modification of this method has been carried out, which allows for a given accuracy to significantly reduce the time for finding the maximum value. A scheme is given and the operation of this algorithm for optimizing the functional quality of agents' work is considered.

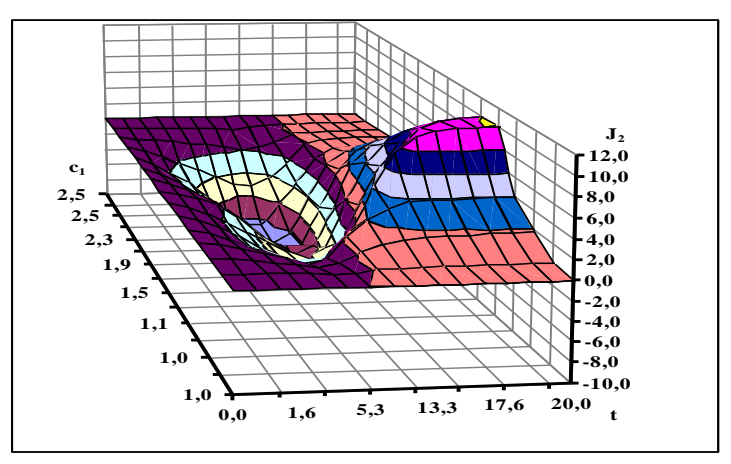

Fig. 8. The Dependence of the Functional J2 on $\mathrm{t}$ and $\mathrm{c} 1$.

\section{CONCLUSIONS}

It was conducted a system analysis of DSS in the management of business processes EGs. This made it possible to determine directions and methods for optimizing their management systems for use in implementing joint business processes. Also by using the Pontryagin maximum principle, the problem of managing the distribution of investments over the step of the implementation of a joint IP for the MAS of the enterprise and agent-investor has been solved. This made it possible to determine the optimal trajectories of changes in the management process of IP, which, unlike the existing ones, takes into account both the initial and final values of the required parameters of the investment process. It is shown that the application of the developed methodology allows the investor to reduce their costs while achieving the same results of the investment process.

Expressions are obtained for the functional quality (objective functions) of the MAS, taking into account both static and dynamic indicators of their joint work. This made it possible to determine the points in time at which management decisions should be made in the DSS to change the parameters characterizing the work of the MAS, depending on the tasks assigned to each agent. A consideration was given to managing dynamic MAS of agent-enterprise and agentinvestor collaboration, taking into account the possible options for the agent-enterprise to use the profit and investment of the agent-investor. It is shown that in order to obtain maximum values of the functional quality for each of the agents from teamwork, they need to change the modes of using their profits (for the enterprise) and foreign investments (for the investor) over time. Expressions are obtained to determine the points in time at which the control parameters of the agents should be changed to ensure the greatest efficiency of the MAS operation. It is also considered the analysis of dynamic systems, consisting of manufacturers and consumers of industrial products for an arbitrary period of time.

A scheme and management options for parallel and sequential work of agents in the DSS, based on a dynamic analysis of the manufacturing processes and sales of industrial products have been developed. Modes and times of changes in control parameters, the duration and nature of changes in the performance of agents in such a system are determined from the condition for each agent to receive the maximum values of the functional quality from their joint work. The initial data of the sequential work of the enterprise have been determined, which enable them to organize continuous work in a closed loop in the manufacture and sale of products. The structure and development of DSS in the management of EGs business processes and structural transformations of EGs has been determined. Approbation and implementation of the DSS was carried out in the state enterprise, confirming the effectiveness and efficiency of its use on real objects.

\section{REFERENCES}

[1] Leach, L.P., Critical chain project management. 2014: Artech House.

[2] AlRababah, A.A., A. AlShahrani, and B. Al-Kasasbeh, Efficiency Model of Information Systems as an Implementation of Key Performance Indicators. International Journal of Computer Science and Network Security (IJCSNS), 2016. 16(12): p. 139. 
[3] Othman, S.B., et al., An agent-based decision support system for resources' scheduling in emergency supply chains. Control Engineering Practice, 2017. 59: p. 27-43.

[4] Chan, S.H., et al., Decision support system (DSS) use and decision performance: DSS motivation and its antecedents. Information \& Management, 2017. 54(7): p. 934-947.

[5] Goodwin, P. and G. Wright, Decision Analysis for Management Judgment 5th ed. 2014: John Wiley and sons.

[6] Noe, R.A., et al., Human resource management: Gaining a competitive advantage. 2017: McGraw-Hill Education New York, NY.

[7] Rose, D.C., et al., Involving stakeholders in agricultural decision support systems: Improving user-centred design. International Journal of Agricultural Management, 2018. 6(3-4): p. 80-89.

[8] Ruiz, P.A.P., B. Kamsu-Foguem, and D. Noyes, Knowledge reuse integrating the collaboration from experts in industrial maintenance management. Knowledge-Based Systems, 2013. 50: p. 171-186.

[9] de Souza Melaré, A.V., et al., Technologies and decision support systems to aid solid-waste management: a systematic review. Waste management, 2017. 59: p. 567-584.

[10] Garousi, V., et al. Industry-academia collaborations in software engineering: An empirical analysis of challenges, patterns and antipatterns in research projects. in 21st International Conference on Evaluation and Assessment in Software Engineering (EASE 2017). 2017. ACM.

[11] Van Huben, G.A. and J.L. Mueller, Data management system for file and database management. 2000, Google Patents.
[12] Al-Rababah, A. and N. Hani. Component linked based system. in Modern Problems of Radio Engineering, Telecommunications and Computer Science, 2004. Proceedings of the International Conference. 2004. IEEE.

[13] Al-rababah, A.A. and M.A. Al-rababah, Module Management Tool in Software Development Organizations 1. 2007.

[14] 14. Sahir, S.H., R. Rosmawati, and R. Rahim, Fuzzy model tahani as a decision support system for selection computer tablet. Int. J. Eng. Technol, 2018. 7(2.9): p. 61-65.

[15] Al-Rababah, A.A., T. AlTamimi, and N. Shalash, A New Model for Software Engineering Systems Quality Improvement. Research Journal of Applied Sciences, Engineering and Technology, 2014. 7(13): p. 27242728.

[16] Caniëls, M.C. and R.J. Bakens, The effects of Project Management Information Systems on decision making in a multi project environment. International Journal of Project Management, 2012. 30(2): p. 162-175.

[17] Al-Rababah, A.A. and M.A. Al-Rababah, Functional Activity Based Comparison Study for Neural Network Application. IJCSNS, 2007. 7(1): p. 153.

[18] Al Ofeishat, H.A. and A.A. Al-Rababah, Real-time programming platforms in the mainstream environments. IJCSNS, 2009. 9(1): p. 197.

[19] Gupta, P. Accelerating datacenter workloads. in 26th International Conference on Field Programmable Logic and Applications (FPL). 2016.

[20] AlRababah, A., Digital Image Encryption Implementations Based on AES Algorithm. VAWKUM Transactions on Computer Sciences, 2017. 13(1): p. 1-9. 